\title{
Moving Frames and Differential Invariants in Centro-Affine Geometry
}

\author{
Peter J. Olver ${ }^{\dagger}$ \\ School of Mathematics \\ University of Minnesota \\ Minneapolis, MN 55455 \\ olver@math . umn . edu \\ http: //www. math. umn.edu/ olver
}

\begin{abstract}
Explicit formulas for the generating differential invariants and invariant differential operators for curves in two- and three-dimensional centro-equi-affine and centroaffine geometry and surfaces in three-dimensional centro-equi-affine geometry are constructed using the equivariant method of moving frames. In particular, the algebra of centro-equi-affine surface differential invariants is shown to be generated by a single second order invariant.
\end{abstract}

$\dagger$ Supported in part by NSF Grant DMS 08-07317.

March 30, 2010 


\section{Introduction.}

In this paper, the equivariant method of moving frames developed in $[\mathbf{2}, \mathbf{1 4}]$ will be applied to study the differential invariants of curves and surfaces in two- and threedimensional space under the standard representations of the special linear and general linear groups. In geometric language, these are referred to as centro-equi-affine geometry or, alternatively, unimodular centro-affine geometry, and centro-affine geometry, respectively. Centro-affine geometry has its origins in the work of the early twentieth century Romanian mathematician Tzitzeica, and was developed in depth by a number of his compatriots; see [1]. Earlier treatments of invariants of curves and hypersurfaces in centro-affine geometry using the classical Cartan approach to moving frames can be found in $[\mathbf{3}, \mathbf{1 0}, \mathbf{1 5}, \mathbf{1 6}]$. Applications to ordinary differential equations can be found in $[\mathbf{6}, \mathbf{1 7}]$, and to control theory in [18]. This particular investigation was sparked by correspondence with Peter Giblin in connection with his collaboration with Takashi Sano on height and distance functions in Klein geometries, [4].

In the case of plane curves, there is a single generating differential invariant, the centro-(equi-)affine curvature. Moreover, a complete system of functionally independent differential invariants can be obtained by differentiating the curvature invariant with respect to the centro-(equi-)affine arc length. For curves in three-dimensional space, there are two independent generating differential invariants, the centro-(equi-)affine curvature and torsion. Again, differentiating these two invariants with respect to the centro-(equi-)affine arc length produces all the higher order invariants.

In the case of surfaces in three-dimensional space, there is a single second order centroequi-affine differential invariant. Moreover, we prove that the algebra of surface differential invariants can, in fact, be generated by this single differential invariant, in the sense that all the higher order differential invariants can be obtained by repeatedly applying the two invariant differential operators associated with the moving frame to the generating invariant and taking functional combinations thereof. This is reminiscent of similar surprising recent results in Euclidean and equi-affine surface geometry, [12], as well as conformal and projective geometry, $[7]$.

\section{Plane Curves.}

We begin by studying plane curves $C \subset \mathbb{R}^{2}$. We are interested in the standard linear action

$$
X=\alpha x+\beta u, \quad U=\gamma x+\delta u
$$

of the general linear group $\mathrm{GL}(2)$ on $\mathbb{R}^{2}$, so that

$$
g=\left(\begin{array}{cc}
\alpha & \beta \\
\gamma & \delta
\end{array}\right) \in \operatorname{GL}(2), \quad \alpha \delta-\beta \gamma \neq 0 .
$$

However, before analyzing the full linear action, we will restrict our attention to unimodular or area-preserving linear maps, i.e., consider the action of the special linear group $\mathrm{SL}(2) \subset$ $\mathrm{GL}(2)$ that is given by (2.1) along with the restriction

$$
g=\left(\begin{array}{cc}
\alpha & \beta \\
\gamma & \delta
\end{array}\right) \in \mathrm{SL}(2), \quad \alpha \delta-\beta \gamma=1 .
$$


We will denote points in the plane by $z=(x, u) \in \mathbb{R}^{2}$. Consider the induced action of $\mathrm{SL}(2)$ on parametrized curves

$$
z(t)=(x(t), u(t)) \text {, where the parameter } t \text { lies in an open subinterval } t \in I \subset \mathbb{R} .
$$

To construct a (right) equivariant moving frame, we prolong the action (2.1) to the curve jet spaces $\mathrm{J}^{n}=\mathrm{J}^{n}(M, 1)$, defined as the bundle of equivalence classes of curves under the equivalence relation of $n^{\text {th }}$ order contact, cf. [11]. We use the standard local coordinates $z^{(n)}=\left(x, u, u_{x}, u_{x x}, \ldots u_{n}\right)$ on $\mathrm{J}^{n}$. Assuming that the curve (2.4) intersects the vertical fibers transversally ${ }^{\dagger}$, which requires $x_{t} \neq 0$, its $n$-jet $\mathrm{j}_{n} C \subset \mathrm{J}^{n}$ is parametrized by the differential functions

$$
u_{x}=D_{x} u=\frac{u_{t}}{x_{t}}, \quad u_{x x}=D_{x} u_{x}=\frac{x_{t} u_{t t}-u_{t} x_{t t}}{x_{t}^{3}}, \quad u_{x x x}=D_{x} u_{x x}, \quad \ldots \quad u_{n}=D_{x} u_{n-1},
$$

where the $t$ subscripts denote derivatives with respect to the curve parameter, while

$$
D_{x}=\frac{1}{x_{t}} \frac{d}{d t}
$$

is the total derivative operator on $\mathrm{J}^{n}$.

The explicit expressions for the prolonged action of GL(2) on $\mathrm{J}^{n}$ induced by (2.1) are obtained by implicit differentiation. The transformed horizontal form ${ }^{\ddagger}$ is

$$
d X=\left(\alpha x_{t}+\beta u_{t}\right) d t
$$

with dual implicit differentiation operator

$$
D_{X}=\frac{1}{\alpha x_{t}+\beta u_{t}} \frac{d}{d t} .
$$

Thus, the jet coordinates of the transformed curve $\bar{C}=g \cdot C$, where $g \in \mathrm{GL}(2)$, are obtained by repeatedly applying (2.8) to $v$ as given in the second equation of (2.1):

$$
U_{X}=D_{X} U=\frac{\gamma x_{t}+\delta u_{t}}{\alpha x_{t}+\beta u_{t}}, \quad U_{X X}=D_{X} U_{X}=(\alpha \delta-\beta \gamma) \frac{x_{t} u_{t t}-u_{t} x_{t t}}{\left(\alpha x_{t}+\beta u_{t}\right)^{3}},
$$

and so on. In the unimodular case, we also impose the constraint (2.3).

The existence of an equivariant moving frame requires that the prolonged group act freely, meaning that the isotropy subgroup at any jet is trivial, [2]. The prolonged action of $\mathrm{SL}(2)$ is free on the dense open subset

$$
\mathcal{V}=\left\{0 \neq z \wedge z_{t}=x u_{t}-u x_{t}\right\} \subset \mathrm{J}^{1}
$$

$\dagger$ If not, one can use a change of coordinate, e.g., by reversing $x$ and $u$. Moreover, the differential invariants that we ultimately derive will be applicable to any smooth curve.

$\ddagger$ Here, we ignore contact components, which vanish when evaluated on curve jets. However, further applications to the invariant calculus of variations, [9], and to invariant curve flows, [13], would necessitate keeping track of the contact forms. 
consisting of jets of curves at a point $z$ that are not tangent to the line connecting $z$ to the origin. The moving frame construction is based on a (local) cross-section $K \subset \mathcal{V}$ to the prolonged group orbits, and we select the particular cross-section defined by the equations

$$
x=0, \quad u=1, \quad u_{x}=0 .
$$

The solution to the associated normalization equations

$$
X=0, \quad U=1, \quad U_{X}=0,
$$

will define a right-equivariant moving frame. Using (2.9) and (2.3), we find that

$$
\alpha=u, \quad \beta=-x, \quad \gamma=\frac{-u_{t}}{u x_{t}-x u_{t}}, \quad \delta=\frac{x_{t}}{u x_{t}-x u_{t}},
$$

which, as the reader can check, does define a right equivariant map $\rho: \mathcal{V} \rightarrow \operatorname{SL}(2)$.

To obtain the differential invariants, we invariantize the higher order jet coordinates, $[\mathbf{2}]$, - that is, substitute the moving frame formulae (2.12) into the prolonged transformation rules (2.5). In particular, the lowest order differential invariant comes from

$$
U_{X X} \longmapsto \frac{x_{t} u_{t t}-u_{t} x_{t t}}{\left(u x_{t}-x u_{t}\right)^{3}}=-\frac{z_{t} \wedge z_{t t}}{\left(z \wedge z_{t}\right)^{3}}=-\kappa,
$$

which we identify as minus the centro-equi-affine curvature invariant, [3]. Here

$$
z_{1} \wedge z_{2}=x_{1} u_{2}-x_{2} u_{1}, \quad z_{1}=\left(x_{1}, u_{1}\right), \quad z_{2}=\left(x_{2}, u_{2}\right),
$$

denotes the usual (scalar-valued) cross product between vectors in the plane. The centroequi-affine arc length element is obtained by invariantizing the horizontal form (2.7):

$$
d X \longmapsto\left(u x_{t}-x u_{t}\right) d t=-\left(z \wedge z_{t}\right) d t=-d s .
$$

Higher order differential invariants are obtained by successively differentiating the curvature $\kappa$ with respect to arc length; the first of these is the third order differential invariant

$$
\kappa_{s}=\frac{\left(z \wedge z_{t}\right)\left(z_{t} \wedge z_{t t t}\right)-3\left(z \wedge z_{t t}\right)\left(z_{t} \wedge z_{t t}\right)}{\left(z \wedge z_{t}\right)^{5}} .
$$

If we parametrize the curve using the centro-equi-affine arc length parameter $s$, then the formulae simplify as follows. Note first that $s$ satisfies

$$
z \wedge z_{s}=1, \quad \text { and hence } \quad z \wedge z_{s s}=0, \quad z \wedge z_{s s s}=-z_{s} \wedge z_{s s} .
$$

Therefore, the first two differential invariants are simply

$$
\kappa=z_{s} \wedge z_{s s}, \quad \kappa_{s}=z_{s} \wedge z_{s s s} .
$$

Incidentally, the corresponding left-equivariant moving frame $\widehat{\rho}: \mathcal{V} \rightarrow \operatorname{SL}(2)$ is obtained by inverting the right-equivariant frame. The result is simply $\widehat{\rho}\left(z^{(1)}\right)=\left(z_{s}, z\right)$, i.e., the columns of the matrix consist of the centro-equi-affine tangent along with the point on the curve, the former being fixed by the requirement that the parallelogram spanned 
by these two vectors has unit area. This reconfirms the observation that the geometrically based moving frames are always left-equivariant, [2]. The associated Frenet equation, [5], is

$$
z_{s s}=-\kappa z
$$

For the full affine group GL(2), one can proceed, ab initio, in a similar fashion. An alternative is to apply Kogan's inductive construction, [8] based on the $\mathrm{SL}(2)$ moving frame given above. If only the differential invariants are desired, the simplest approach is to determine how the SL(2) invariants behave under a scaling map

$$
z \longmapsto \lambda z
$$

and then to take suitably invariant rational combinations thereof. Clearly, under (2.20),

$$
\kappa \longmapsto \lambda^{-4} \kappa, \quad d s \longmapsto \lambda^{2} d s, \quad \kappa_{s} \longmapsto \lambda^{-6} \kappa_{s} .
$$

Therefore, the lowest order centro-affine differential invariant is

$$
\varkappa=\frac{\kappa_{s}}{\kappa^{3 / 2}}=\frac{\left(z \wedge z_{t}\right)\left(z_{t} \wedge z_{t t t}\right)-3\left(z \wedge z_{t t}\right)\left(z_{t} \wedge z_{t t}\right)}{\left(z \wedge z_{t}\right)^{1 / 2}\left(z_{t} \wedge z_{t t}\right)^{3 / 2}}
$$

which we identify as the centro-affine curvature, defined for curves satisfying the nondegeneracy conditions

$$
z \wedge z_{t} \neq 0, \quad z_{t} \wedge z_{t t} \neq 0
$$

Similarly, the centro-affine arc length is

$$
d \sigma=\sqrt{\kappa} d s=\sqrt{\frac{z_{t} \wedge z_{t t}}{z \wedge z_{t}}} d t
$$

The condition that the curve is parametrized with respect to centro-affine arc length is

$$
\left(z_{\sigma \sigma}-z\right) \wedge z_{\sigma}=0
$$

In this case, the centro-equi-affine curvature is given by

$$
\kappa=\frac{1}{\left(z \wedge z_{\sigma}\right)^{2}}
$$

and therefore the centro-affine curvature (2.22) is

$$
\varkappa=\frac{\kappa_{\sigma}}{\kappa}=-2 \frac{z \wedge z_{\sigma \sigma}}{z \wedge z_{\sigma}} .
$$

In this case, the Frenet equation takes the form

$$
z_{\sigma \sigma}=z-\frac{1}{2} \varkappa z_{\sigma}
$$




\section{Space Curves.}

We now turn our attention to the centro-(equi)-affine geometry of curves in threedimensional space: $C \subset \mathbb{R}^{3}$. We will denote points in space by $z=(x, u, v) \in \mathbb{R}^{3}$, with $z(t)=(x(t), u(t), v(t))$ for $t \in I \subset \mathbb{R}$ being a smoothly parametrized curve.

We consider the usual linear action

$$
(X, U, V)=Z=g \cdot z=g \cdot(x, u, v)
$$

of the general linear group $\mathrm{GL}(3)$ on $\mathbb{R}^{3}$, so that

$$
X=\alpha \cdot z, \quad U=\beta \cdot z, \quad V=\gamma \cdot z,
$$

where $\alpha, \beta, \gamma$, are the rows of the $3 \times 3$ matrix

$$
g=\left(\begin{array}{l}
\alpha \\
\beta \\
\gamma
\end{array}\right) \in \operatorname{GL}(3), \quad \operatorname{det} g=[\alpha, \beta, \gamma]=\alpha \cdot \beta \wedge \gamma \neq 0 .
$$

We use $[\alpha, \beta, \gamma]$ to denote the matrix determinant, or, equivalently, the vector triple product of its rows, with $\wedge$ denoting the vector cross product in $\mathbb{R}^{3}$.

Before tacking the full linear action, we first specialize to volume-preserving linear maps, and so restrict the action (3.1) to the special linear group

$$
g=\left(\begin{array}{l}
\alpha \\
\beta \\
\gamma
\end{array}\right) \in \operatorname{SL}(3), \quad \text { so that } \quad \operatorname{det} g=[\alpha, \beta, \gamma]=\alpha \cdot \beta \wedge \gamma=1 .
$$

To construct a (right) equivariant moving frame, we prolong the $\mathrm{SL}(3)$ action $(3.1,3)$ to the curve jet spaces $\mathrm{J}^{n}=\mathrm{J}^{n}(M, 1)$, which has local coordinates

$$
z^{(n)}=\left(x, u, v, u_{x}, v_{x}, u_{x x}, v_{x x}, \ldots u_{n}, v_{n}\right),
$$

whose expressions for a general parametrized curve are more or less the same as in (2.5). As before, the formulae for the prolonged action on $\mathrm{J}^{n}$ are provided by implicit differentiation, based on

$$
d X=\left(\alpha \cdot z_{t}\right) d t, \quad D_{X}=\frac{1}{\alpha \cdot z_{t}} \frac{d}{d t} .
$$

The first few are

$$
U_{X}=D_{X} U=\frac{\beta \cdot z_{t}}{\alpha \cdot z_{t}}, \quad U_{X X}=D_{X} U_{X}=\frac{\left(\alpha \cdot z_{t}\right)\left(\beta \cdot z_{t t}\right)-\left(\beta \cdot z_{t}\right)\left(\alpha \cdot z_{t t}\right)}{\left(\alpha \cdot z_{t}\right)^{3}}
$$

while $U_{X X X}=D_{X} U_{X X}$ equals

$$
\frac{\left(\alpha \cdot z_{t}\right)^{2}\left(\beta \cdot z_{t t t}\right)-\left(\alpha \cdot z_{t}\right)\left(\beta \cdot z_{t}\right)\left(\alpha \cdot z_{t t t}\right)-3\left(\alpha \cdot z_{t}\right)\left(\alpha \cdot z_{t t}\right)\left(\beta \cdot z_{t t}\right)+3\left(\beta \cdot z_{t}\right)\left(\alpha \cdot z_{t t}\right)^{2}}{\left(\alpha \cdot z_{t}\right)^{3}} .
$$


We will also require the even longer formula for the transformation of $u_{x x x x}$, but this will not be written out here. The expressions for the transformed $v_{x}, v_{x x}, \ldots$, are obtained by replacing $\beta$ by $\gamma$ in the preceding formulae.

We need to specify $\operatorname{dim} \mathrm{SL}(3)=8$ cross-section equations, which we choose to be

$$
x=0, \quad u=0, \quad v=1, \quad u_{x}=0, \quad v_{x}=0, \quad u_{x x}=1, \quad v_{x x}=0, \quad u_{x x x}=0 .
$$

The associated normalization equations are obtained by replacing the jet coordinates in the cross-section equation by their transformed versions $X, U, V, U_{X}, \ldots$, cf. $(3.1,5,6)$. After some obvious simplification, we find

$$
\begin{aligned}
\alpha \cdot z=0, & \alpha \cdot z_{t}=\lambda, & 3 \lambda^{2} \alpha \cdot z_{t t}=\lambda^{2} \beta \cdot z_{t t t}, \\
\beta \cdot z=0, & \beta \cdot z_{t}=0, & \beta \cdot z_{t t}=\lambda^{2}, \\
\gamma \cdot z=1, & \gamma \cdot z_{t}=0, & \gamma \cdot z_{t t}=0,
\end{aligned}
$$

with $\lambda$ an as yet unspecified scalar. Let us set

$$
\Delta=\left[z, z_{t}, z_{t t}\right] \quad \text { so that } \quad \Delta_{t}=\left[z, z_{t}, z_{t t t}\right] .
$$

We impose the nondegeneracy condition $\Delta \neq 0$, meaning that the osculating plane to the curve is transverse to the line connecting the origin to the point on the curve. The second and third row of equations in (3.8) immediately imply

$$
\beta=\frac{\lambda^{2} z \wedge z_{t}}{\Delta}, \quad \gamma=\frac{z_{t} \wedge z_{t t}}{\Delta}
$$

The first normalization equation implies that

$$
\alpha=\mu z \wedge z_{t}+\nu z \wedge z_{t t}
$$

for certain scalars $\mu, \nu$. Substituting these expressions into the second and third equations produces the formulae

$$
\mu=\frac{\lambda \Delta_{t}}{3 \Delta^{2}}, \quad \nu=-\frac{\lambda}{\Delta}
$$

Finally, substituting the expressions for $\alpha, \beta$ and $\gamma$ in the unimodularity constraint, we find

$$
1=[\alpha, \beta, \gamma]=-\frac{\lambda^{3}}{\Delta^{3}}\left[z \wedge z_{t t}, z \wedge z_{t}, z_{t} \wedge z_{t t}\right]=\frac{\lambda^{3}}{\Delta}, \quad \text { and hence } \quad \lambda=\Delta^{1 / 3}
$$

where we make use of the elementary determinantal identity

$$
\left[z \wedge z_{t t}, z \wedge z_{t}, z_{t} \wedge z_{t t}\right]=-\left[z, z_{t}, z_{t t}\right]^{2}=-\Delta^{2} .
$$

Therefore, the right-equivariant moving frame induced by the cross-section (3.7) is given by

$$
\alpha=\frac{\Delta_{t} z \wedge z_{t}}{3 \Delta^{5 / 3}}-\frac{z \wedge z_{t t}}{\Delta^{2 / 3}}, \quad \beta=\frac{z \wedge z_{t}}{\Delta^{1 / 3}}, \quad \gamma=\frac{z_{t} \wedge z_{t t}}{\Delta} .
$$

As always, a complete system of functionally independent differential invariants is obtained by invariantization, that is, substituting the moving frame formulae (3.11) into the 
unnormalized transformation rules. The generating differential invariants are the centroequi-affine curvature $\kappa$, obtained from $v_{x x x}$, and the centro-equi-affine torsion $\tau$, obtained from $u_{x x x x}$. All other differential invariants are obtained by differentiating the curvature and torsion with respect to the centro-equi-affine arc length element

$$
d s=\Delta^{1 / 3} d t
$$

which comes from substituting the moving frame formula for $\alpha$ into (3.4). A short computation produces the required expressions:

$$
\begin{aligned}
& \kappa=\frac{\left[z_{t}, z_{t t}, z_{t t t}\right]}{\Delta^{2}}, \\
& \tau=\frac{\left[z, z_{t}, z_{t t t t}\right]+4\left[z, z_{t t}, z_{t t t}\right]}{\Delta^{5 / 3}}-\frac{5 \Delta_{t}^{2}}{3 \Delta^{8 / 3}}=-\frac{3}{2} \frac{d^{2}}{d t^{2}}\left(\frac{1}{\Delta^{2 / 3}}\right)+\frac{3\left[z, z_{t t}, z_{t t t}\right]}{\Delta^{5 / 3}} .
\end{aligned}
$$

Parametrization of the curve with respect to centro-equi-affine arc length requires

$$
\Delta=\left[z, z_{s}, z_{s s}\right]=1,
$$

leading to the following simplified expressions for the curvature and torsion invariants:

$$
\kappa=\left[z_{s}, z_{s s}, z_{s s s}\right], \quad \tau=3\left[z, z_{s s}, z_{s s s}\right] .
$$

Inverting the matrix whose rows are the right moving frame vectors (3.11) produces the corresponding geometric left-equivariant moving frame ${ }^{\dagger} \widehat{\rho}\left(z^{(3)}\right)=\left(z_{s}, z_{s s}, z\right) \in \mathrm{SL}(3)$. The associated Frenet equation, [5], is

$$
z_{s s s}=\kappa z-\frac{1}{3} \tau z_{s}
$$

Turning our attention to the full centro-affine action, under scaling,

$$
z \longmapsto \lambda z, \quad \kappa \longmapsto \lambda^{-3} \kappa, \quad \tau \longmapsto \lambda^{-2} \tau, \quad d s \longmapsto \lambda d s .
$$

Therefore, the lowest order GL(3) differential invariants are

$$
\widehat{\kappa}=\frac{\kappa_{s}}{\kappa^{4 / 3}}, \quad \widehat{\tau}=\frac{\tau}{\kappa^{2 / 3}},
$$

which we identify as the centro-affine curvature and torsion - although in this case they are both fourth order differential invariants. Similarly, the centro-affine arc length is

$$
d \sigma=\kappa^{1 / 3} d s=\sqrt[3]{\frac{\left[z_{t}, z_{t t}, z_{t t t}\right]}{\left[z, z_{t}, z_{t t}\right]}} d t
$$

The condition that the curve is parametrized with respect to centro-affine arc length is

$$
\left[z_{\sigma \sigma \sigma}-z, z_{\sigma}, z_{\sigma \sigma}\right]=0
$$

$\dagger$ Here, the $z$ 's are viewed as column vectors. 
In this case, the centro-equi-affine curvature is given by

$$
\kappa=\frac{1}{\left[z, z_{\sigma}, z_{\sigma \sigma}\right]}=\frac{1}{\Delta} .
$$

Therefore the centro-affine curvature and torsion are

$$
\begin{aligned}
& \widehat{\kappa}=\frac{\kappa_{\sigma}}{\kappa}=-\frac{\left[z, z_{\sigma}, z_{\sigma \sigma \sigma}\right]}{\left[z, z_{\sigma}, z_{\sigma \sigma}\right]} \\
& \widehat{\tau}=-\frac{\kappa_{\sigma \sigma}}{\kappa}+\frac{\kappa_{\sigma}^{2}}{3 \kappa^{2}}+3 \kappa\left[z, z_{\sigma \sigma}, z_{\sigma \sigma \sigma}\right]=-\widehat{\kappa}_{\sigma}+\frac{4}{3} \widehat{\kappa}^{2}+3 \frac{\left[z, z_{\sigma \sigma}, z_{\sigma \sigma \sigma}\right]}{\left[z, z_{\sigma}, z_{\sigma \sigma}\right]} .
\end{aligned}
$$

Thus, we can instead use the simpler expressions

$$
\widetilde{\kappa}=\frac{\left[z, z_{\sigma}, z_{\sigma \sigma \sigma}\right]}{\left[z, z_{\sigma}, z_{\sigma \sigma}\right]}, \quad \widetilde{\tau}=\frac{\left[z, z_{\sigma \sigma}, z_{\sigma \sigma \sigma}\right]}{\left[z, z_{\sigma}, z_{\sigma \sigma}\right]},
$$

as the fundamental centro-affine differential invariants for space curves.

\section{Surfaces.}

We now turn our attention to the centro-equi-affine geometry of surfaces in threedimensional space: $S \subset M=\mathbb{R}^{3}$. In this case, we denote points in space by $z=(x, y, u) \in$ $\mathbb{R}^{3}$, with $z(s, t)=(x(s, t), y(s, t), u(s, t))$ being a parametrized surface. We impose the usual regularity condition

$$
z_{s} \wedge z_{t} \neq 0
$$

on the underlying surface parametrization. We consider the same linear action of SL(3), that maps a point $z=(x, y, u) \in \mathbb{R}^{3}$ to the image point $Z=(X, Y, U)$, with

$$
X=\alpha \cdot z, \quad Y=\beta \cdot z, \quad U=\gamma \cdot z,
$$

where $\alpha, \beta, \gamma$ continue to satisfy (3.3).

To construct a (right) equivariant moving frame, we prolong the action (4.2) to the surface jet spaces $\mathrm{J}^{n}=\mathrm{J}^{n}(M, 2)$, with local coordinates

$$
z^{(n)}=\left(x, y, u, u_{x}, u_{y}, u_{x x}, u_{x y}, u_{y y}, \ldots, u_{j k}, \ldots\right), \quad j+k \leq n,
$$

where $u_{j k}$ indicates the derivative $\partial^{j+k} u / \partial x^{j} \partial y^{k}$. As before, the formulae for the prolonged action on $\mathrm{J}^{n}$ are provided by implicit differentiation with respect to the transformed horizontal one-forms

$$
d X=\alpha_{s} d s+\alpha_{t} d t, \quad d Y=\beta_{s} d s+\beta_{t} d t .
$$

where, to make the formulas less cluttered in the sequel, we abbreviate

$$
\alpha_{s}=\alpha \cdot z_{s}, \quad \alpha_{t}=\alpha \cdot z_{t}, \quad \alpha_{t t}=\alpha \cdot z_{t t}, \quad \ldots
$$

and similarly for $\beta, \gamma$. Keep in mind that, unlike those on $z$, the subscripts on $\alpha, \beta, \gamma d o$ not indicate derivatives, but rather the scalars obtained by taking dot products with the corresponding derivatives of $z$ with respect to the surface parameters. 
The dual implicit differentiations to the transformed one-forms (4.3) are, therefore,

$$
D_{X}=\frac{1}{\delta}\left(\beta_{t} D_{s}-\beta_{s} D_{t}\right), \quad D_{Y}=\frac{1}{\delta}\left(-\alpha_{t} D_{s}+\alpha_{s} D_{t}\right),
$$

where

$$
\delta=\alpha_{s} \beta_{t}-\alpha_{t} \beta_{s}=\left(\alpha \cdot z_{s}\right)\left(\beta \cdot z_{t}\right)-\left(\alpha \cdot z_{t}\right)\left(\beta \cdot z_{s}\right) .
$$

Note that $\delta$ is a scalar. Consequently, the first prolonged transformations are given by

$$
U_{X}=\frac{\beta_{t} \gamma_{s}-\beta_{s} \gamma_{t}}{\delta}, \quad U_{Y}=\frac{\alpha_{s} \gamma_{t}-\alpha_{t} \gamma_{s}}{\delta} .
$$

Let us also write one of the second order formulas:

$$
\begin{aligned}
U_{X X}= & \frac{\beta_{t}\left(\beta_{t} \gamma_{s s}+\beta_{t s} \gamma_{s}-\beta_{s} \gamma_{s t}-\beta_{s s} \gamma_{t}\right)-\beta_{s}\left(\beta_{t} \gamma_{s t}+\beta_{t t} \gamma_{s}-\beta_{s} \gamma_{t t}-\beta_{s t} \gamma_{t}\right)}{\delta^{2}}- \\
& -\frac{\beta_{t} \delta_{s}-\beta_{s} \delta_{t}}{\delta^{2}} U_{X},
\end{aligned}
$$

the rest having similar expressions that the reader can easily find.

Interestingly, even though $\operatorname{dim} \mathrm{SL}(3)=8$ and $\operatorname{dim} \mathrm{J}^{2}=8$, the prolonged action is not (even locally) transitive. Indeed, the orbits in $\mathrm{J}^{2}$ are, generically, seven-dimensional, and thus there is a second order differential invariant! The easiest way to deduce this is to look at the prolonged infinitesimal generators. Recall, [11], that the $n^{\text {th }}$ prolongation of a vector field

$$
\mathbf{v}=\xi(x, y, u) \frac{\partial}{\partial x}+\eta(x, y, u) \frac{\partial}{\partial y}+\varphi(x, y, u) \frac{\partial}{\partial u}
$$

on $\mathbb{R}^{3}$ is the vector field

$$
\mathbf{v}^{(n)}=\mathbf{v}+\sum_{1 \leq j+k \leq n} \varphi^{j k}\left(x, y, u^{(j+k)}\right) \frac{\partial}{\partial u_{j k}}
$$

on $\mathrm{J}^{n}=\mathrm{J}^{n}\left(\mathbb{R}^{3}, 2\right)$, whose coefficients are given by

$$
\varphi^{j k}=D_{x}^{j} D_{y}^{k}\left(\varphi-\xi u_{x}-\eta u_{y}\right)+\xi u_{j+1, k}+\eta u_{k, j+1} .
$$

For the linear action of SL(3), a basis for the second order prolonged infinitesimal generators is provided by the eight vector fields

$$
\begin{aligned}
& x \partial_{x}-u \partial_{u}-2 u_{x} \partial_{u_{x}}-u_{y} \partial_{u_{y}}-3 u_{x x} \partial_{u_{x x}}-2 u_{x y} \partial_{u_{x y}}-u_{y y} \partial_{u_{y y}}, \\
& y \partial_{y}-u \partial_{u}-u_{x} \partial_{u_{x}}-2 u_{y} \partial_{u_{y}}-u_{x x} \partial_{u_{x x}}-2 u_{x y} \partial_{u_{x y}}-3 u_{y y} \partial_{u_{y y}}, \\
& y \partial_{x}-u_{x} \partial_{u_{y}}-u_{x x} \partial_{u_{x y}}-2 u_{x y} \partial_{u_{y y}}, \\
& u \partial_{x}-u_{x}^{2} \partial_{u_{x}}-u_{x} u_{y} \partial_{u_{y}}-3 u_{x} u_{x x} \partial_{u_{x x}}-\left(u_{y} u_{x x}+2 u_{x} u_{x y}\right) \partial_{u_{x y}}-\left(2 u_{y} u_{x y}+u_{x} u_{y y}\right) \partial_{u_{y y}}, \\
& x \partial_{y}-u_{y} \partial_{u_{x}}-2 u_{x y} \partial_{u_{x x}}-u_{y y} \partial_{u_{x y}} \\
& u \partial_{y}-u_{x} u_{y} \partial_{u_{x}}-u_{y}^{2} \partial_{u_{y}}-\left(u_{y} u_{x x}+2 u_{x} u_{x y}\right) \partial_{u_{x x}}-\left(2 u_{y} u_{x y}+u_{x} u_{y y}\right) \partial_{u_{x y}}-3 u_{y} u_{y y} \partial_{u_{y y}}, \\
& x \partial_{u}-\partial_{u_{x}} \\
& y \partial_{u}-\partial_{u_{y}} .
\end{aligned}
$$


For generic $z^{(2)} \in \mathrm{J}^{2}$, they span a 7-dimensional subspace of $\left.T \mathrm{~J}^{2}\left(\mathbb{R}^{3}, 2\right)\right|_{z^{(2)}}$, thereby proving the claim.

We will eventually prove, using moving frame methods, the following result, which is very much in the spirit of those established in [12] and [7].

Theorem 4.1. All differential invariants of a generic centro-equi-affine surface $S \subset$ $\mathbb{R}^{3}$ are generated by a single second order differential invariant through invariant differentiation. Namely,

$$
I=H / \Delta^{2}
$$

where

$$
H=\left[z_{s}, z_{t}, z_{s t}\right]^{2}-\left[z_{s}, z_{t}, z_{s s}\right]\left[z_{s}, z_{t}, z_{t t}\right], \quad \Delta=\left[z, z_{s}, z_{t}\right] .
$$

In particular, when $S$ coincides with the graph of the function $u=u(x, y)$,

$$
H=u_{x y}^{2}-u_{x x} u_{y y}, \quad \Delta=u-x u_{x}-y u_{y},
$$

so that $H$ reduces to minus the Hessian of $u$. In this case, the generating differential invariant is simply

$$
I=\frac{u_{x y}^{2}-u_{x x} u_{y y}}{\left(u-x u_{x}-y u_{y}\right)^{2}} .
$$

Since the prolonged action is not free on $\mathrm{J}^{2}$, the lowest order moving frame will be of third order. We will work with the "hyperbolic" local cross-section:

$$
x=0, \quad y=0, \quad u=1, \quad u_{x}=u_{y}=u_{x x}=u_{y y}=0, \quad u_{x x x}=1 .
$$

The fact that this defines a local cross-section follows from the fact that the third order prolonged infinitesimal generators are linearly independent at each jet belonging to it. As we will see, the resulting moving frame will be defined for hyperbolic surfaces, that satisfy $I>0$. For brevity, we will not discuss the elliptic case, where $I<0$, although it is straightforward to deduce the corresponding differential invariants and their interrelationships from our computations. The parabolic case $I \equiv 0$ requires a higher order moving frame.

To construct the hyperbolic moving frame, we solve the corresponding normalization equations

$$
X=0, \quad Y=0, \quad U=1, \quad U_{X}=U_{Y}=U_{X X}=U_{Y Y}=0, \quad U_{X X X}=1,
$$

for the group parameters. In view of (4.2), the first three require that

$$
\alpha \cdot z=0, \quad \beta \cdot z=0, \quad \gamma \cdot z=1 .
$$

Using (4.7), the following two normalization equations for the first order derivatives, imply

$$
\alpha_{t} \gamma_{s}=\alpha_{s} \gamma_{t}, \quad \beta_{t} \gamma_{s}=\beta_{s} \gamma_{t} .
$$

I claim that this requires both

$$
0=\gamma_{s}=\gamma \cdot z_{s}, \quad 0=\gamma_{t}=\gamma \cdot z_{t} .
$$


Indeed, suppose $\gamma_{s} \neq 0$. Then (4.18) implies that

$$
\alpha_{t}=\lambda \alpha_{s}, \quad \beta_{t}=\lambda \beta_{s}, \quad \gamma_{t}=\lambda \gamma_{s},
$$

for some scalar $\lambda$. But, in view of (4.4), these conditions are equivalent to

$$
\alpha \cdot\left(z_{t}-\lambda z_{s}\right)=\beta \cdot\left(z_{t}-\lambda z_{s}\right)=\gamma \cdot\left(z_{t}-\lambda z_{s}\right)=0 .
$$

Thus, in view of (4.1), the vector

$$
z_{t}-\lambda z_{s} \neq 0
$$

is a non-zero element of kernel of the matrix $g \in \mathrm{SL}(3)$ with rows $\alpha, \beta, \gamma$, which is a contradiction. Thus $\gamma_{s}=0$ and, by a similar argument, $\gamma_{t}=0$ also. Combining (4.19) with the third equation in (4.17), we deduce that

$$
\gamma=\frac{z_{s} \wedge z_{t}}{\Delta}, \quad \text { where } \quad \Delta=\left[z, z_{s}, z_{t}\right] \neq 0 .
$$

The latter nondegeneracy condition requires that the tangent plane to the surface at the point $z \in S$ be transverse to the vector from the origin to $z$.

We next look at the second order normalization equations $U_{X X}=U_{Y Y}=0$. Using (4.8) and its counterpart for $U_{Y Y}$, and keeping (4.19) in mind, we find that

$$
\alpha_{t}^{2} \gamma_{s s}-2 \alpha_{s} \alpha_{t} \gamma_{s t}+\alpha_{s}^{2} \gamma_{t t}=0, \quad \beta_{t}^{2} \gamma_{s s}-2 \beta_{s} \beta_{t} \gamma_{s t}+\beta_{s}^{2} \gamma_{t t}=0
$$

Note that (4.21) implies that

$\gamma_{s s}=\gamma \cdot z_{s s}=\frac{\left[z_{s}, z_{t}, z_{s s}\right]}{\Delta}, \quad \gamma_{s t}=\gamma \cdot z_{s t}=\frac{\left[z_{s}, z_{t}, z_{s t}\right]}{\Delta}, \quad \gamma_{t t}=\gamma \cdot z_{t t}=\frac{\left[z_{s}, z_{t}, z_{t t}\right]}{\Delta}$.

Consider the quadratic equation

$$
\gamma_{s s} r^{2}-2 \gamma_{s t} r+\gamma_{t t}=0
$$

and let

$$
\begin{aligned}
& r_{1}=\frac{\gamma_{s t}-\sqrt{\gamma_{s t}^{2}-\gamma_{s s} \gamma_{t t}}}{\gamma_{s s}}=\frac{\left[z_{s}, z_{t}, z_{s t}\right]-\sqrt{H}}{\left[z_{s}, z_{t}, z_{s s}\right]} \\
& r_{2}=\frac{\gamma_{s t}+\sqrt{\gamma_{s t}^{2}-\gamma_{s s} \gamma_{t t}}}{\gamma_{s s}}=\frac{\left[z_{s}, z_{t}, z_{s t}\right]+\sqrt{H}}{\left[z_{s}, z_{t}, z_{s s}\right]}
\end{aligned}
$$

be its two roots, which are real in the hyperbolic regime $I=H / \Delta^{2}>0$. Then (4.22) implies that either

$$
\alpha_{t}-r_{1} \alpha_{s}=\alpha \cdot\left(z_{t}-r_{1} z_{s}\right)=0 \quad \text { and } \quad \beta_{t}-r_{2} \beta_{s}=\beta \cdot\left(z_{t}-r_{2} z_{s}\right)=0,
$$

or the equations obtained by reversing $r_{1} \leftrightarrow r_{2}$. Indeed, these two equations must involve different roots, as otherwise, when combined with (4.19), this would lead to the same contradiction as in (4.20). For specificity, we will stick with the situation given above during the rest of the computation. Combining (4.26) with (4.17), we find

$$
\alpha=\mu z \wedge\left(z_{t}-r_{1} z_{s}\right), \quad \beta=\nu z \wedge\left(z_{t}-r_{2} z_{s}\right),
$$


for certain scalars $\mu, \nu$. Furthermore,

$$
\alpha_{s}=-\mu \Delta, \quad \alpha_{t}=-\mu r_{1} \Delta, \quad \beta_{s}=-\nu \Delta, \quad \beta_{t}=-\nu r_{2} \Delta .
$$

Note that this implies that the quantity (4.6) is given simply by

$$
\delta=\mu \nu\left(r_{2}-r_{1}\right) \Delta^{2}=\frac{2 \mu \nu \Delta^{2} \sqrt{H}}{\left[z_{s}, z_{t}, z_{s s}\right]} .
$$

Now, even though we as yet don't have the complete moving frame, we can still derive the second order differential invariant by substituting our formulas $(4.21,28,29)$ for $\alpha, \beta, \gamma$ and $\delta$ into that of $U_{X Y}$. The non-vanishing terms are

$$
U_{X Y}=\frac{-\alpha_{t} \beta_{t} \gamma_{s s}+\left(\alpha_{s} \beta_{t}+\alpha_{t} \beta_{s}\right) \gamma_{s t}-\alpha_{s} \beta_{s} \gamma_{t t}}{\delta^{2}}=\frac{\left[z_{s}, z_{t}, z_{s s}\right]}{2 \mu \nu \Delta^{2}} .
$$

On the other hand, the unimodularity constraint $(3.3)$ coupled with $(4.21,27)$ implies

$$
\begin{aligned}
1=[\alpha, \beta, \gamma] & =\frac{\mu \nu}{\Delta}\left[z_{s} \wedge z_{t}, z \wedge\left(z_{t}-r_{1} z_{s}\right), z \wedge\left(z_{t}-r_{2} z_{s}\right)\right] \\
& =\mu \nu \Delta\left(r_{2}-r_{1}\right)=\frac{2 \mu \nu \Delta \sqrt{H}}{\left[z_{s}, z_{t}, z_{s s}\right]}
\end{aligned}
$$

by a slight generalization of the determinantal identity (3.10). Therefore,

$$
\mu \nu=\frac{\left[z_{s}, z_{t}, z_{s s}\right]}{2 \Delta \sqrt{H}}, \quad \text { and so } \quad \delta=\Delta .
$$

Substituting this into (4.30) produces the second order differential invariant $\sqrt{H} / \Delta=\sqrt{I}$. In other words, the differential invariant (4.13) is minus the square of the invariantization of the jet coordinate $u_{x y}$ with respect to the moving frame specified by the cross-section (4.15).

The two invariant differentiation operators are obtained by normalizing the implicit differentiation operators (4.5), and so require us to implement the final moving frame normalization in (4.16). We calculate the explicit formula for the third order transformed jet coordinate $U_{X X X}$ by directly applying the implicit differentiation operator $D_{X}$ to (4.8). After substituting the moving frame normalizations - specifically (4.19) — the remaining nonzero terms are

$$
\begin{aligned}
& 1=\delta^{-3}\left[\beta_{t}^{3} \gamma_{s s s}-3 \beta_{s} \beta_{t}^{2} \gamma_{s s t}+3 \beta_{s}^{2} \beta_{t} \gamma_{s t t}-\beta_{s}^{3} \gamma_{t t t}+\right. \\
& \left.\quad+3\left(\beta_{t}^{2} \beta_{s t}-\beta_{s} \beta_{t} \beta_{t t}\right) \gamma_{s s}+3\left(\beta_{t}^{2} \beta_{s s}-\beta_{s}^{2} \beta_{t t}\right) \gamma_{s t}+3\left(\beta_{s} \beta_{t} \beta_{s s}-\beta_{s}^{2} \beta_{s t}\right) \gamma_{s s}\right] .
\end{aligned}
$$

We then substitute $(4.21,23,28,31)$, and solve resulting equation for

$$
\nu=\sqrt[3]{\Delta / J}
$$

where

$$
\begin{array}{r}
J=-\left[z_{s}, z_{t}, r_{2}^{3} z_{s s s}+r_{2}^{2} z_{s s t}+r_{2} z_{s t t}+z_{t t t}\right]+3\left[z_{s}, z_{t}, z_{s s}\right]\left[z, z_{t}-r_{2} z_{s}, r_{2} z_{t t}+r_{2}^{2} z_{s t}\right]+ \\
+3\left[z_{s}, z_{t}, z_{s t}\right]\left[z, z_{t}-r_{2} z_{s}, z_{t t}-r_{2}^{2} z_{s s}\right]-3\left[z_{s}, z_{t}, z_{t t}\right]\left[z, z_{t}-r_{2} z_{s}, z_{s t}+r_{2} z_{s s}\right] .
\end{array}
$$


Thus, the hyperbolic centro-equi-affine moving frame is given by

$$
\alpha=\frac{\left[z_{s}, z_{t}, z_{s s}\right] J^{1 / 3}}{2 \Delta^{4 / 3} \sqrt{H}} z \wedge\left(z_{t}-r_{1} z_{s}\right), \quad \beta=\frac{\Delta^{1 / 3}}{J^{1 / 3}} z \wedge\left(z_{t}-r_{2} z_{s}\right), \quad \gamma=\frac{z_{s} \wedge z_{t}}{\Delta}
$$

Substituting these expressions into the implicit differentiation operators (4.5) produces the invariant differential operators $\mathcal{D}_{1}, \mathcal{D}_{2}$.

$$
\mathcal{D}_{1}=\frac{\Delta^{1 / 3}}{J^{1 / 3}}\left(r_{2} D_{s}+D_{t}\right), \quad \mathcal{D}_{2}=-\frac{\left[z_{s}, z_{t}, z_{s s}\right] J^{1 / 3}}{2 \Delta^{4 / 3} \sqrt{H}}\left(r_{1} D_{s}+D_{t}\right) .
$$

The proof that the second order differential invariant $I$ generate the entire differential invariant algebra through iterated invariant differentiation relies on methods introduced in [12] and further developed in [7]. We will assume the reader of this last part is familiar with these references, and so will be content to outline the computations, which are based on the fundamental moving frame recurrence formulae, $[\mathbf{2}]$, without detailed justification.

We let

$$
I_{j k}=\iota\left(u_{j k}\right), \quad j, k \geq 0
$$

denote the normalized differential invariants obtained by invariantization of the surface jet coordinates. In particular, the phantom invariants resulting from the choice of cross-section are

$$
I_{00}=1, \quad I_{10}=I_{01}=I_{20}=I_{02}=0, \quad I_{30}=1,
$$

while

$$
I_{11}=\iota\left(u_{x y}\right)=\sqrt{I}
$$

is the square root of the second order differential invariant (4.13). There are three independent, non-constant differential invariants of order 3 , namely $I_{21}, I_{12}, I_{03}$, and five more of order 4 , namely $I_{40}, I_{31}, I_{22}, I_{13}, I_{04}$. Since our moving frame is of order 3 , a general theorem, [2], (which is a consequence of the recurrence formulae below), implies that one can generate all the higher order differential invariants by invariant differentiation of the 9 normalized differential invariants of order $\leq 4$, that is, one more than the order of the moving frame. Thus, to establish Theorem 4.1 we need only show how to express the third and fourth order differential invariants as combinations of invariant derivatives of $I_{11}$.

To accomplish this, we make use of the explicit recurrence formulae. The key result, $[\mathbf{7}, \mathbf{1 2}]$, is the following:

Theorem 4.2. The recurrence formulae for the differentiated invariants are

$$
\begin{aligned}
& \mathcal{D}_{1} I_{j k}=I_{j+1, k}+\sum_{\kappa=1}^{8} \varphi_{\kappa}^{j k}\left(0,0, I^{(j+k)}\right) R_{1}^{\kappa}, \\
& \mathcal{D}_{2} I_{j k}=I_{j, k+1}+\sum_{\kappa=1}^{8} \varphi_{\kappa}^{j k}\left(0,0, I^{(j+k)}\right) R_{2}^{\kappa},
\end{aligned}
$$

where $R_{i}^{\kappa}$ are certain differential invariants, known as the Maurer-Cartan invariants, while $\varphi_{\kappa}^{j k}\left(0,0, I^{(j+k)}\right)=\iota\left(\varphi_{\kappa}^{j k}\left(x, y, u^{(j+k)}\right)\right)$ is the invariantization of the coefficient of $\partial / \partial u_{j k}$ in the prolonged infinitesimal generator $\mathbf{v}_{\kappa}$, as given in (4.11). 
The Maurer-Cartan invariants are, in fact, uniquely determined from the recurrence formulae for the phantom differential invariants (4.36), which, being constant, give recurrence formulae (4.38) with vanishing left hand sides. The result is a linear algebraic system for the Maurer-Cartan invariants, and a direct computation produces their formulas in terms of the normalized differential invariants (4.35):

$$
R=\left(R_{i}^{\kappa}\right)=\left(\begin{array}{cc}
\frac{1}{3} I_{40}-\frac{1}{2} I_{21} / I_{11} & \frac{1}{3} I_{31}-\frac{1}{2} I_{21}^{2} / I_{11} \\
\frac{1}{2} I_{12} / I_{11} & \frac{1}{2} I_{03} / I_{11} \\
-1 & 0 \\
\frac{1}{2} / I_{11} & \frac{1}{2} I_{21} / I_{11} \\
-\frac{1}{3} I_{40}+\frac{1}{2} I_{21} / I_{11} & -\frac{1}{3} I_{31}+\frac{1}{2} I_{21}^{2} / I_{11} \\
0 & -1 \\
0 & -I_{11} \\
-I_{11} & 0
\end{array}\right) .
$$

The other ingredient is the commutator formula between the invariant differential operators, which has the form

$$
\left[\mathcal{D}_{1}, \mathcal{D}_{2}\right]=\mathcal{D}_{1} \mathcal{D}_{2}-\mathcal{D}_{2} \mathcal{D}_{1}=Y_{1} \mathcal{D}_{1}+Y_{2} \mathcal{D}_{2}
$$

where, by the methods of $[\mathbf{2}]$, the commutator invariants are

$$
Y_{1}=\frac{1}{3} I_{31}-\frac{I_{21}^{2}+I_{12}}{2 I_{11}}, \quad Y_{2}=\frac{1}{3} I_{40} .
$$

The commutator trick of [12] allows us to express both commutator invariants as certain rational combinations of derivatives of $I_{11}$. Indeed, applying (4.40) to $I_{11}$ and any one of its derivatives, say $\mathcal{D}_{1} I_{11}$, leads to a pair of linear algebraic equations whose coefficients depend on $I_{11}$ and its invariant derivatives. These linear equations can be immediately solved for $Y_{1}, Y_{2}$, producing the desired formulae.

With these in hand, we analyze selected non-phantom recurrence formulae (4.38). First, setting $j=k=1$,

$$
\mathcal{D}_{1} I_{11}=I_{21}, \quad \mathcal{D}_{2} I_{11}=I_{12},
$$

immediately gives two of the third order invariants. Now, since we can write the commutator invariants (4.41) and $I_{21}, I_{12}$ in terms of $I_{11}$, the same holds for $I_{40}, I_{31}$. Next, subtracting the recurrence formulae

$$
\begin{aligned}
& \mathcal{D}_{1} I_{31}=I_{41}-\frac{2}{3} I_{40} I_{31}+4 I_{11}-\frac{I_{12} I_{40}-2 I_{21} I_{31}+3 I_{22}}{2 I_{11}}, \\
& \mathcal{D}_{2} I_{40}=I_{41}-\frac{4}{3} I_{40} I_{31}+4 I_{11}-2 \frac{I_{21}^{2} I_{40}-I_{21} I_{31}}{I_{11}},
\end{aligned}
$$

and then replacing $I_{40}, I_{31}, I_{21}, I_{12}$ by our already established formulae, we deduce a formula for $I_{22}$ in terms of $I_{11}$. Then, using

$$
\mathcal{D}_{2} I_{21}=I_{22}-\frac{1}{3} I_{21} I_{31}+2 I_{11}^{2}+\frac{I_{21}^{3}-2 I_{21} I_{12}-I_{03}}{2 I_{11}},
$$


we obtain a formula for the remaining third order differential invariant $I_{03}$. The last two fourth order differential invariants, $I_{13}, I_{04}$ can then be obtained by differentiating $I_{03}$ :

$$
\mathcal{D}_{1} I_{03}=I_{13}+I_{03} I_{40}-\frac{3\left(I_{12}^{2}+I_{21} I_{03}\right)}{2 I_{11}}, \quad \mathcal{D}_{2} I_{03}=I_{04}+I_{03} I_{31}-\frac{3\left(I_{21}^{2}+I_{12}\right) I_{03}}{2 I_{11}} .
$$

This completes the proof of Theorem 4.1.

The corresponding moving frame analysis of centro-affine surfaces will, in the interests of brevity, be deferred until a subsequent paper.

Acknowledgments: It is a pleasure to thank Peter Giblin for inspiring me to write this paper, and for helpful advice during its genesis. 


\section{References}

[1] Cruceanu, V., Research works of Romanian mathematicians on centro-affine geometry, Balkan J. Geom. Appl. 10 (2005), 1-5.

[2] Fels, M., and Olver, P.J., Moving coframes. II. Regularization and theoretical foundations, Acta Appl. Math. 55 (1999), 127-208.

[3] Gardner, R.B., and Wilkens, G.R., The fundamental theorems of curves and hypersurfaces in centro-affine geometry, Bull. Belg. Math. Soc. Simon Stevin 4 (1997), 379-401.

[4] Giblin, P.J., and Sano, T., personal communication, 2008-9.

[5] Guggenheimer, H.W., Differential Geometry, McGraw-Hill, New York, 1963.

[6] Guggenheimer, H.W., Hill equations with coexisting periodic solutions, J. Diff. Eq. 5 (1969), 159-166.

[7] Hubert, E., and Olver, P.J., Differential invariants of conformal and projective surfaces, SIGMA 3 (2007), 097.

[8] Kogan, I.A., Inductive construction of moving frames, Contemp. Math. 285 (2001), $157-170$.

[9] Kogan, I.A., and Olver, P.J., Invariant Euler-Lagrange equations and the invariant variational bicomplex, Acta Appl. Math. 76 (2003), 137-193.

[10] Mayer, O., and Myller, A., La géométrie centroaffine des courbes planes, Ann. Sci. Univ. Jassy 18 (1933), 234-280.

[11] Olver, P.J., Equivalence, Invariants, and Symmetry, Cambridge University Press, Cambridge, 1995.

[12] Olver, P.J., Differential invariants of surfaces, Diff. Geom. Appl. 27 (2009), 230-239.

[13] Olver, P.J., Invariant submanifold flows, J. Phys. A 41 (2008), 344017.

[14] Olver, P.J., Lectures on moving frames, in: Symmetries and Integrability of Difference Equations, Levi, D., Olver, P., Thomova, Z., and Winternitz, P., eds., Cambridge University Press, Cambridge, to appear.

[15] Pekşen, Ö., and Khadjiev, D., On invariants of curves in centro-affine geometry, $J$. Math. Kyoto Univ. 44 (2004), 603-613.

[16] Schirokow, A.P., and Schirokow, P.A., Affine Differentialgeometrie, B.G. Teubner, Leipzig, 1962. (German translation of Russian original)

[17] Sibirsky, K.S., Introduction to the Algebraic Theory of Invariants of Differential Equations, Manchester University Press, New York, 1988.

[18] Wilkens, G.R., Centro-affine geometry in the plane and feedback invariants of two-state scalar control systems, in: Differential Geometry and Control, G. Ferreyra, R. Gardner, H. Hermes and H. Sussmann, eds., Proc. Sympos. Pure Math., vol. 64, Amer. Math. Soc., Providence, R.I., 1999, pp. 319-333. 